\title{
Desempenho financeiro e valor de mercado do setor de telefonia no Brasil
}

\section{Financial performance and market value sector telephony in Brazil}

\author{
Carolina Terra Resende Silva ${ }^{1}$ \\ David Ferreira Lopes Santos ${ }^{2}$
}

\section{Resumo}

O trabalho proposto estudou a relação entre métricas tradicionais de desempenho financeiro e o impacto no valor de mercado das empresas de telefonia do Brasil presentes na BM\&FBOVESPA entre 2002 e 2012. Construiu-se uma base de dados com informações trimestrais das quatro empresas com informações completas no período (TIM, Vivo, Embratel e Oi), sendo esta base analisada por meio de regressão com dados em painel. Os resultados assinalam que os indicadores tradicionais de desempenho financeiro ROS, ROI, ROE, Margem EBTIDA não foram capazes de explicar a variação no valor de mercado, tampouco as métricas de valor agregado EVA, MVA e RROI. Encontraram-se como significativos na explicação do valor de mercado destas empresas o Investimento realizado e o custo médio ponderado de capital (WACC).

Palavras-chave: Valor de Mercado. Desempenho Financeiro. Setor de Telefonia.

\section{Abstract}

This study investigated the relationship between traditional metrics of financial performance and the impact on the market value of the telephony companies in the Brazil, with stock in BM\&FBOVESPA between 2002 and 2012. Constructed a database containing financial statements of the four companies with complete information for the period (TIM, Vivo, Embratel and Oi), which is based analyzed by regression using panel data method. The results indicate that traditional indicators of financial performance ROS, ROI, ROE, EBITDA margin were not able to explain the variation in market value, either, the metrics of value added EVA, MVA and

Graduada em Administração pela UNESP - Universidade Estadual Paulista Júlio de Mesquita Filho.carol_trs@hotmail.com

2 Professor Assistente Doutor na UNESP junto ao Departamento de Economia Rural da Faculdade de Ciências Agrárias e Veterinárias. david.lopes@fcav.unesp.br 
RROI. In explaining the market value of these companies the Investment and WACC (Weighted Capital Average Cost) were found as significant.

Keywords: Market Value. Financial Performance. Sector Telephony.

\section{Introdução}

Dentro de um ambiente competitivo e com informações simétricas o valor de mercado de uma empresa deve refletir a sua capacidade criação de riqueza, no entanto, os métodos de valoração são diversos e as premissas que os sustentam são flexíveis para comportar a idiossincrasia de cada analista ou investidor (DAMODARAN, 2001; GRAHAM; HARVEY, 2001).

O mercado observa com cuidado os fundamentos econômicos e financeiros das empresas, de modo que os indicadores de desempenho e medidas de criação de valor constituem-se um dos principais instrumentos de controle quanto às condições e capacidade de longevidade das empresas (O'BYRNE, 1996; KRAUTER; SOUSA, 2007; JUNG, 2008).

Diante da atual conjuntura em que o foco maior das empresas está na necessidade de criar valor para os acionistas e demais stakeholders, setores regulamentados e com alto grau de competitividade demandam uma gestão voltada para o valor de mercado (WHEATLEY, 1988; LI; WHALLEY, 2002; CUÉLLAR-FERNANDÉZ; FUERTES-CALLÉN; LAÍNEZ-GADEA, 2011; GODOY; MORALES, 2012). O setor de telefonia brasileiro pode ser disposto neste contexto devido aos índices de crescimento, uma vez que somente em 2012 foram registrados 19,54 milhões de novas habilitações de telefonia móvel totalizando acréscimo de $8,07 \%$ na base de assinantes, além de ser conhecido pela sua competitividade (ANATEL, 2013).

Portanto, o objetivo deste estudo é avaliar a relação entre o desempenho financeiro e o valor de mercado do setor de telefonia do Brasil. Espera-se que a compreensão das premissas que influenciam o valor de mercado dessas empresas contribua para um melhor entendimento das práticas e condições financeiras deste setor econômico fundamental para as relações econômicas, empresariais e sociais do país. 
Diferentes pesquisas têm se encarregado de verificar o grau de associação entre métricas de desempenho financeiro com o valor de mercado como Lehn e Makhija (1996), O’Byrne (1996), Biddle, Bowen e Wallace (1997), Krauter (2004), Salvi (2007), Paiva, Savoia e Sousa (2008), Bastos et al. (2009) e Xin, Ting e Yuan (2012). No entanto, o setor de telefonia ainda não foi objeto de análise desta relação, em especial, no Brasil.

Desta forma, além de lançar luzes sobre este setor, o estudo trabalhou um modelo quantitativo de análise, a partir do uso de regressão com dados em um painel equilibrado. Essa abordagem permitiu analisar a relação entre o desempenho financeiro e o valor de mercado das quatro maiores empresas de telefonia no Brasil com capital aberto na BM\&FBOVESPA durante dez anos. Com efeito, tem-se o primeiro estudo no Brasil sobre o tema proposto para este setor.

\section{Referencial teórico}

\subsection{Desempenho financeiro e valor de mercado}

O desempenho empresarial contempla três domínios, cujos graus são crescentes em abrangência. O primeiro é o desempenho financeiro, o segundo o desempenho operacional e, por fim, a eficácia organizacional (BRITO; BRITO; MORGANTI, 2009).

Nesse trabalho, o escopo é analisar a relação do desempenho financeiro no valor de mercado, sendo que analisar financeiramente uma empresa significa transformar dados contábeis em informações importantes para direcionar as decisões de gestores e analistas quanto aos objetivos da organização (GODOY; MORALES, 2012).

Para fazer a mensuração do desempenho é necessária a utilização de métricas, ou seja, indicadores de desempenho. As medidas tradicionais de mensuração do desempenho possuem a vantagem de fácil disponibilidade em base de dados (BARCELLOS, 2010; XIN; TING; YUAN, 2012). 
Krauter e Sousa (2007) utilizam-se dos estudos de Young e O'Byrne (2001) para demonstrar uma classificação das medidas de desempenho separando-as em cinco grupos como demonstrados no Quadro 1.

Quadro 1: Classificação das medidas de avaliação de desempenho financeiro

\begin{tabular}{|c|c|c|}
\hline Grupo & Descrição & Exemplos \\
\hline $\begin{array}{l}\text { Medidas de } \\
\text { lucro residual }\end{array}$ & $\begin{array}{l}\text { Cobram pelo custo } \\
\text { de capital (próprio } \\
\text { e de terceiros). Não } \\
\text { inclui oportunidades e } \\
\text { crescimento futuro do } \\
\text { mercado. }\end{array}$ & $\begin{array}{l}\text { i) Cash Value Added (CVA) } \\
\text { ii) Lucro Econômico } \\
\left.\text { iii) Economic Value Added (EVA }{ }^{\circledast}\right)\end{array}$ \\
\hline $\begin{array}{l}\text { Componentes } \\
\text { do lucro } \\
\text { residual }\end{array}$ & $\begin{array}{l}\text { Elementos do lucro que } \\
\text { não incluem os custos } \\
\text { do capital. Utilização } \\
\text { comum em níveis } \\
\text { inferiores da estrutura } \\
\text { organizacional. }\end{array}$ & $\begin{array}{l}\text { iv) Earnings Before Interest and } \\
\text { Taxes (EBIT) } \\
\text { v) Earnings Before Interest, Taxes, } \\
\text { Depreciation and Amortization } \\
\text { (EBTIDA) } \\
\text { vi) Net Operating Profit After Taxes } \\
\text { (NOPAT) } \\
\text { vii) Return On Net Assets (RONA) }\end{array}$ \\
\hline $\begin{array}{l}\text { Medidas } \\
\text { baseadas no } \\
\text { mercado }\end{array}$ & $\begin{array}{l}\text { Derivadas do mercado } \\
\text { de capital. Portanto, } \\
\text { somente são disponíveis } \\
\text { para empresas de } \\
\text { capital aberto cujas } \\
\text { ações são negociadas } \\
\text { no mercado. } \\
\end{array}$ & $\begin{array}{l}\text { viii) Total Shareholder Return (TSR) } \\
\text { ix) Market Value Added (MVA) }\end{array}$ \\
\hline $\begin{array}{l}\text { Medidas de } \\
\text { fluxo de caixa }\end{array}$ & \begin{tabular}{l|} 
São utilizadas para \\
contornar a influência \\
do regime de \\
competência utilizado na \\
contabilidade.
\end{tabular} & $\begin{array}{l}\text { x) Fluxo de Caixa das Operações } \\
\text { xi) Fluxo de Caixa Livre para } \\
\text { acionistas } \\
\text { xii) Cash Flow Return on Investment } \\
\text { (CFROI) }\end{array}$ \\
\hline $\begin{array}{l}\text { Medidas } \\
\text { de lucro } \\
\text { tradicionais }\end{array}$ & $\begin{array}{l}\text { Medidas que executivos } \\
\text { e analistas tem utilizado } \\
\text { há décadas. }\end{array}$ & $\begin{array}{l}\text { xiii) Lucro Líquido } \\
\text { xiv) Lucro por Ação }\end{array}$ \\
\hline
\end{tabular}

Fonte: Adaptado Krauter e Sousa (2007, p. 3) 
Entretanto, um estudo anterior realizado por Basso, Alves e Nakamura (2002) com profissionais do mercado retrata as dez medidas de desempenho mais utilizadas no Brasil, sendo: $1^{\circ}$ Retorno sobre o Investimento (ROI); $2^{\circ}$ Retorno sobre o Patrimônio Líquido (ROE); $3^{\circ}$ Lucro por Ação (LPA); $4^{\circ}$ Retorno do Ativo Total (ROA); $5^{\circ}$ Índice Preço/ Lucro (P/L); $6^{\circ}$ Retorno sobre os Ativos Líquidos (RONA).

As métricas de rentabilidade medem, sobretudo, retornos. Com eles, é possível avaliar o lucro da empresa em relação ao nível de vendas, de ativos ou também o volume de capital investido pelos proprietários ou total de recursos destinados aos investimentos. Entre eles estão o ROA (Retorno sobre o Ativo), o ROE (Retorno sobre o Patrimônio Líquido), ROS (Retorno sobre a receita) e o ROI (Retorno sobre o Investimento) (GITMAN, 2004; XIN; TING; YUAN, 2012).

Nessediapasão, osindicadores dedesempenhomais desenvolvidos em análise financeira também são enumerados pelos autores tradicionais nas seções inicias do estudo de finanças corporativas (ASSAF NETO, 2005; GITMAN, 2007; ROSS; WESTERFIELD; JORDAN, 2008).

\subsection{Valor de Mercado}

Segundo Kayo et al. (2006), duas considerações importantes devem ser feitas ao se avaliar o valor econômico de uma empresa: o valor intrínseco e o valor de mercado. O primeiro se refere ao método de fluxo de caixa descontado, uma vez que identifica o fluxo de caixa futuro esperado pelo investidor. O segundo representa o valor de mercado da empresa, o qual é resultado da soma do valor de mercado das ações e do valor de mercado das dívidas que reflete diariamente as expectativas dos investidores frente às informações disponíveis ao mercado.

Teoricamente, o valor de mercado deveria igualar o valor intrínseco, porém, devido às ineficiências do mercado de capitais, raramente esses valores se equivalem (DAMODARAN, 2001; KAYO et al., 2006). O valor de mercado geralmente diverge do valor de investimento da empresa, segundo Srinivasan e Hanssens (2009), pois o preço das ações está 
amplamente relacionado com à expectativa futura dos investidores a partir dos resultados empresariais observados, fazendo com que os valores sofram constantes mudanças (BASTOS et al., 2009; MEDEIROS, 2009). Dessa forma, fica evidenciada a necessidade de identificar quais os indicadores de desempenho utilizados pelos analistas influenciam o valor de mercado.

Segundo Medeiros (2009) e Caixe (2012), o conceito dos termos lucro residual e econômico teria surgido há cerca de dois séculos por Hamilton (1777) e posteriormente por Marshall (1890). No entanto, a medida para criação de valor, conhecida como valor econômico agregado (VEA ou o EVA $^{\circledR}$ ), só foi desenvolvida por Bernnett G. Stewart na década de 1980 e constitui juntamente com o Market Value Added (MVA) os principais indicadores de mensuração de valor (BIDDLE; BOWESON; WALLACE, 1997; BASSO, 2003; DENARDIN, 2004). De acordo Stewart III (1990), ao calcular o EVA ${ }^{\circledR}$ para uma empresa é possível determinar o valor criado ou destruído em cada período para o acionista.

As fórmulas para cálculo do $E \mathrm{~A}^{\circledR}$ podem ser encontradas de diversas maneiras e que entregam o mesmo resultado como, por exemplo, a equação 1 (ASSAF NETO, 2005; PEREZ; FAMÁ, 2006; GODOY; MORALES, 2012; XIN; TING; YUAN, 2012; SANTOS; LEITE, 2013).

$E V A=(R O I-W A C C) \times$ Investimentos

Em que:

Weighted Average Cost of Capital (WACC) ou Custo Médio Ponderado de Capital (CMPC)

Investimento $=$ capital total (fixo e de giro) aplicado na empresa Retorno sobre o Investimento (ROI)

O ROI é o resultado do produto do giro do investimento e da margem operacional, enquanto que o WACC representa o retorno mínimo exigido pelos credores e acionistas. Ele está relacionado com o custo oportunidade, o qual identifica o sacrifício de remuneração que uma pessoa ou empresa sofre por aplicar seus recursos em um 
investimento alternativo, porém com semelhante risco (O'BYRNE, 1996; BASSO, 2003; GODOY; MORALES, 2012).

Do ponto de vista da teoria econômica, o custo de oportunidade ocorre quando se opta por uma alternativa de ação em detrimento de outras viáveis e mutuamente exclusivas. Assim, o custo oportunidade pode ser identificado como sendo o benefício desprezado dos outros investimentos pela escolha de um determinado (DENARDIN, 2004; JUNG, 2008).

A expressão (ROI - WACC) identificada na fórmula (1) pode também ser representada como Residual Return on Investiment (RROI) ou ROI Residual ou Spread, a qual representa a quantidade do retorno dos investimentos que superou as expectativas dos credores e acionistas (PEREZ; FAMÁ, 2006; LEITE; SANTOS, 2013)

A criação de valor está associada à qualidade das estratégicas empresariais implementadas de modo que o valor criado pela organização e sua cadeia de valor produza retornos suficientes para remunerar os custos de oportunidade dos proprietários de capital (BASSO, 2003).

Múltiplas pesquisas foram realizadas na tentativa de identificar qual a melhor ferramenta utilizada para explicar o desempenho da empresa e o retorno de ações (O'BYRNE, 1996; KRAUTER, 2004; SALVI, 2007; MEDEIROS, 2009). No entanto, não há ainda um consenso sobre quais medidas (EVA ${ }^{\circledR}$, MVA ou as medidas de desempenhos financeiros tradicionais) exercem maior influência sobre o valor de mercado da empresa.

A bibliografia estudada enumera diversos destes estudos, os quais relacionaram o valor de mercado, o VVA $^{\circledR}$ e o MVA como demonstra o Quadro 2: 


\section{Quadro 2: Estudos sobre valor de mercado, EVA ${ }^{\circledR}$ e MVA}

\begin{tabular}{|c|c|c|}
\hline Autores/ Ano & Variáveis independentes & Resultado \\
\hline $\begin{array}{l}\text { Lehn; Makhija } \\
\text { (1996) }\end{array}$ & $\begin{array}{l}\text { Valor Econômico Agregado } \\
\left(\text { EVA }^{\circledR}\right) ; \text { Valor de Mercado } \\
\text { Agregado (MVA); Retorno } \\
\text { sobre o Ativo (ROA); Retorno } \\
\text { sobre Patrimônio Líquido } \\
\text { (ROE) e Retorno sobre a } \\
\text { Receita (ROS). }\end{array}$ & $\begin{array}{l}\text { A correlação do } \text { EVA }^{\circledR} \text { e MVA com o } \\
\text { retorno das ações é positiva. Apesar de } \\
\text { pequena, é superior a correlação com } \\
\text { medidas de desempenho tradicionais } \\
\text { (ROA, ROS e ROE). }\end{array}$ \\
\hline O’Byrne (1996) & $\begin{array}{l}\text { Fluxo de Caixa Livre (FCF); } \\
\text { Lucro Operacional Líquido do } \\
\text { Imposto de Renda (NOPAT); } \\
\text { Valor Econômico Agregado } \\
\left(\text { EVA }^{\circledR}\right)\end{array}$ & $\begin{array}{l}\text { EVA }^{\circledR} \text { está sistematicamente ligado ao } \\
\text { valor de mercado, explicando melhor as } \\
\text { alterações do valor de mercado do que } \\
\text { NOPAT e FCF }\end{array}$ \\
\hline $\begin{array}{l}\text { Biddle; Bowen; } \\
\text { Wallace (1997) }\end{array}$ & $\begin{array}{l}\text { EVA }^{\circledR} ; \text { Lucro Residual (RI); } \\
\text { Fluxo de Caixa Operacional } \\
\text { (CFO); Lucro antes de itens } \\
\text { extraordinários (EBEI). }\end{array}$ & $\begin{array}{l}\text { O Lucro antes de itens extraordinários } \\
\text { (EBEI) possui associação mais forte com } \\
\text { o retorno das ações do que o EVA }{ }^{\circledR} \text {, o RI } \\
\text { e o CFO. }\end{array}$ \\
\hline Krauter (2004) & $\begin{array}{l}\text { (ROE); } \quad \text { (ROI); } \quad(\text { ROA); } \\
\text { (RONA); Lucro por ação; } \\
\text { l̂ndice preço/lucro; } \text { EVA }^{\circledR} ; \\
\text { MVA. }\end{array}$ & $\begin{array}{l}\text { Para o sistema de gestão baseada em } \\
\text { valor, o ROI e ROE, que foram os mais } \\
\text { utilizados, não são bons indicadores, uma } \\
\text { vez que são medidas contábeis podendo } \\
\text { ser manipuláveis e incluem somente } \\
\text { custo de capital de terceiros. }\end{array}$ \\
\hline Salvi (2007) & $\begin{array}{l}\text { Lucro Líquido (LL); Fluxo de } \\
\text { Caixa Operacional (FCO); } \\
\text { Lucro Residual (LR); Valor } \\
\text { Econômico Agregado }\left(\mathrm{EVA}^{\circledR}\right) \text {. }\end{array}$ & $\begin{array}{l}\text { Não houve uma forte relação das variáveis } \\
\text { com o retorno das ações. Assim, não foi } \\
\text { possível identificar superioridade clara } \\
\text { entre as variáveis. }\end{array}$ \\
\hline $\begin{array}{l}\text { Paiva; Savoia; } \\
\text { Sousa (2008) }\end{array}$ & $\begin{array}{l}\text { (ROI); Fluxo de caixa livre } \\
\text { para o acionista em relação } \\
\text { ao ativo total ajustado } \\
\text { (CFROI); preço/lucro (P/L); } \\
\text { Preço em relação ao fluxo de } \\
\text { caixa (P/CF); Margem EBIT } \\
\text { e EBITDA; Giro do Ativo; } \\
\text { Alavancagem; Capex/Ativo; } \\
\text { PL Mercado/PL Contábil. }\end{array}$ & $\begin{array}{l}\text { Foram significativos na explicação da } \\
\text { variação do valor da ação os indicadores: } \\
\text { Giro do Ativo; Alavancagem e variação do } \\
\text { valor de mercado para contábil (market- } \\
\text { to-book). }\end{array}$ \\
\hline $\begin{array}{l}\text { Bastos et al. } \\
(2009)\end{array}$ & $\begin{array}{l}\text { Lucro por Ação (LPA); } \\
\text { ROI; EVA }{ }^{\circledR} \text {; Fluxo de Caixa } \\
\text { Operacional (FCO). }\end{array}$ & $\begin{array}{l}\text { O FCO apresentou melhores resultados } \\
\text { na relação com o retorno das ações } \\
\text { seguido do ROI e LPA. O EVA }{ }^{\circledR} \text { foi o } \\
\text { indicador com menor resultado. }\end{array}$ \\
\hline
\end{tabular}

Fonte: Elaborado pelos autores 
Portanto, este estudo se posiciona como mais um esforço em avaliar a relação entre o valor de mercado e o desempenho financeiro, com o diferencial de trabalhar um setor ainda não estudado, porém de grande relevância para o Brasil.

\section{Metodologia}

O estudo realizado foi uma pesquisa descritiva com abordagem quantitativa. A pesquisa descritiva, de acordo com Koche (2012, p.124) "estuda as relações entre duas variáveis ou mais de um dado fenômeno sem manipulá-las". No caso do trabalho, a relação estudada foi entre os indicadores de desempenho financeiro e o valor de mercado.

A despeito do entendimento mais objetivo das variáveis quantitativas, deve-se considerar que estas não refletem a realidade plenamente, e por isso não podem ser aplicados além dos limites do estudo. Outra limitação importante é a não captação dos fatores qualitativos, partindo-se do princípio que os resultados financeiros incorporam ou traduzem todo o esforço empresarial.

\subsection{Material}

O universo de operadores de telecomunicações no Brasil em 2012 possui um total de doze empresas, incluindo os segmentos de telefonia fixa e móvel (ANATEL, 2013). No entanto, a amostra deste estudo contou com apenas quatro empresas, três delas atuantes nos dois segmentos e uma no segmento de telefonia fixa de ligações para longa distância.

A amostra foi delimitada por conveniência com empresas que tiveram sua origem como empresas privadas em 1998, ano da privatização da Telecomunicações Brasileiras S.A. (Sistema Telebrás), a qual constituiu um dos maiores marcos da história da telefonia brasileira. Além disso, são empresas que possuem ações no mercado de capitais no Brasil, o que facilita face à disponibilidade de dados financeiros e, em adição, são informações auditadas, o que aumenta a confiabilidade da base analisada. Assim, as empresas alvo do estudo 
foram: Embratel Participações S.A, Telefônica Brasil S.A. (Vivo), Oi S.A., TIM Participações S.A.

A empresa Americel S.A. (Claro) não entrou na amostra, pois teve o registro cancelado na BM\&FBOVESPA no dia 12 de setembro de 2012, devido à incorporação pela Claro S.A.

Os dados quantitativos foram obtidos por meio do Sistema Economática $^{\circledR}$ no qual foram levantados demonstrativos financeiros trimestrais entre janeiro de 2002 a dezembro de 2012, além da cotação das ações ordinárias das quatro empresas pertencentes ao setor de telecomunicações.

As variáveis utilizadas foram os tradicionais indicadores de desempenho utilizados na literatura que suportou este estudo: Retorno sobre o Patrimônio Líquido (ROE) (2); Retorno sobre o Investimento (ROI) (3); Retorno sobre Vendas (ROS) (4); EBITDA/Receita (5); EVA ${ }^{\circledR}$ (6) e Valor de Mercado da Empresa (VM) (7) e Variação do Valor de Mercado (VVM) (8).

Além dos indicadores citados, também foi utilizado o WACC (9) (JUNG, 2008). Para este, foi necessário o cálculo prévio do custo do capital próprio (Ke), onde se utilizou o Capital Asset Pricing Model (CAPM) (10), tendo em vista, ser o modelo mais tradicional para este fim no Brasil, em que pese suas limitações (ARAÚJO; OLIVEIRA; SILVA, 2012).

Araújo, Oliveira e Silva (2012) ainda assinalam que a taxa de juros Selic e o CDI são as principais variáveis para determinar o custo do ativo livre de risco no Brasil, diante disso, optou-se pela Selic por ser a taxa que remunera as Letras Financeiras do Tesouro (LFT 252 dias). Em razão da volatilidade da BM\&FBOVESPA no período analisado, o prêmio do risco do mercado $\left(R_{m}-R_{f}\right)$ foi negativo, ou seja, a BM\&FBOVESPA entregou retorno inferior à Selic, diante disso, e assim, decidiu-se utilizar o prêmio histórico do mercado financeiro nacional em $6 \%$ a.a. para todas as empresas (ASSAF NETO, 2005). 
Ressalta-se, ainda, que não foram utilizados os parâmetros do mercado americano para o ativo livre de risco (Treasuries Bills) e o prêmio daquele mercado associado com o risco Brasil conforme sugerem Alvim, Costa e Costa (2010), pois existem empresas da amostra que só atuavam no mercado de capital brasileiro.

O beta das empresas $(\beta)$ foi calculado por meio da relação entre a covariância do retorno do ativo com o retorno de mercado e a variância do retorno de mercado utilizando-se os últimos três anos. Já o custo da dívida para o capital de terceiros foi fornecido pela empresa e apenas subtraído por $34 \%$ da alíquota do Imposto de Renda para Pessoa Jurídica e Contribuição Social do Lucro Líquido. O Quadro 3 apresenta as fórmulas utilizadas e o seu sequenciamento.

Quadro 3 - Identidade das variáveis utilizadas na pesquisa

\begin{tabular}{|c|l|c|l|}
\hline Fórmulas & Nr. & & Nr. \\
\hline$R O E=\frac{\text { Lucro Líquido }}{\text { Patrimônio Líquido }}$ & $(2)$ & $V L=$ Patrimônio Líquido + Dívida & $(7)$ \\
\hline$R O I=\frac{\text { Lucro Operacional Líquido }}{\text { Investimento Médio }}$ & $(3)$ & $V V M=\frac{V M_{t}-V M_{t-1}}{V M_{t-1}}$ & $(8)$ \\
\hline ROS $=\frac{\text { Lucro Líquido }}{\text { Receita Líquida }}$ & $(4)$ & $W A C C=K_{e} \times \frac{P L}{P L+D}+K_{d} \times \frac{D}{P L+D}$ & $(9)$ \\
\hline Margem EBTIDA $=\frac{\text { EBTIDA }}{\text { Receita Líquida }}$ & $(5)$ & $K_{e}=R_{f}+\beta\left(R_{m}-R_{f}\right)$ & $(10)$ \\
\hline EVA $=($ ROI - WACC $) \times$ Investimento & $(6)$ & $\beta_{i}=\frac{C O V_{m, i}}{\sigma_{m}^{2}}$ & $(11)$ \\
\hline
\end{tabular}

Fonte: Elaborado pelos autores

O Patrimônio Líquido (PL) e a Dívida (D) foram tomados pelo valor de mercado, e, assim, a quantidade de ações (ON e PN) foi ponderada pelo seu preço quando da divulgação dos resultados trimestrais, bem como o valor das dívidas.

\subsection{Métodos}

O principal método utilizado foi a Análise de Regressão com Dados em Painel, cuja representação genérica é apresentada na equação 12 (GUJARATI, 2006). Além deste, utilizou-se como instrumento de apoio a técnica tradicional de análise correlação para verificar o grau 
de associação entre as variáveis e aumentar a compreensão dos parâmetros da regressão.

$$
Y_{i t}=\beta_{1 i}+\beta_{2} X_{2 i t}+\beta_{3} X_{3 i t}+\cdots+\beta_{n} X_{n i t}+\mu_{i t}
$$

De acordo com Gujarati (2006, p. 513), "nos dados em painel, a mesma unidade de corte transversal é acompanhada ao longo do tempo. Em síntese, os dados em painel têm uma dimensão espacial e outra temporal". O estudo de Salvi (2007) utilizou da Regressão com Dados em Painel para associar desempenho com retorno das ações e os estudos de Bastos et al. (2009) e Leite e Santos (2013) para associar com valor de mercado.

Na equação 12, a variável dependente "Y" é a variação do valor de mercado. A expectativa é que a variação do valor de mercado de cada empresa "i" em cada trimestre "t" seja explicada pelas variáveis explanatórias.

Nos resultados deste estudo, apresentam-se apenas os resultados significativos, tendo em vista as inúmeras combinações testadas com as diferentes métricas de desempenho. Desse modo, a variável $X_{2}$ assumiu o valor do investimento; a variável $X_{3}$, do WACC e a variável $X_{4}$, dos valores de ROI, ROS, ROE e EBTIDA, intercaladamente, isto é, têm-se quatro regressões.

Os coeficientes parciais da regressão $(\beta)$ são fixos no curso do tempo e representam a influência de cada variável explanatória na variável dependente.

Para a verificação do ajuste e diagnóstico do modelo, foram usados os testes $\mathrm{R}^{2}$, Durbin Watson, Estatística F, Heteroscedasticidade, Qui-quadrado para a normalidade e teste para diferenciar o intercepto do grupo (GUJARATI, 2006). A formulação matemática dos testes pode ser consultada nos manuais tradicionais de econometria. Optou-se pela supressão destes no presente documento em vistas do escopo do trabalho e do uso estrito das técnicas sem variações. 


\section{Resultados}

\subsection{Contexto do setor de telefonia no Brasil}

O mercado de telecomunicações no Brasil, tanto fixo como móvel, passou por grandes reestruturações no final da década de 1990. Acompanhando um processo internacional de privatização, com países como EUA, Inglaterra, França, Japão, Alemanha, México, Chile, Argentina, Peru e Bolívia, em 1995 é aprovada a Emenda Constitucional $n^{\circ} 8$, na qual o setor brasileiro de telecomunicação foi aberto à participação de capital privado (ANATEL, 2001; QUINTELLA; COSTA, 2009).

No ano de 1997, ocorreu o fim do monopólio estatal do setor de telefonia e foi aprovada a Lei n. ${ }^{\circ} 9.472$, de 16 de julho de 1997. Esta Lei, conhecida como Lei Geral de Telecomunicações, criou a Agência Nacional de Telecomunicações (Anatel) como órgão regulador e que se constitui de uma autarquia vinculada ao Ministério das Comunicações (ANATEL, 2001).

No dia 29 de junho de 1998, foi realizado um leilão na Bolsa de Valores do Rio de Janeiro, no qual houve a transferência do controle societário anteriormente detido pela União para quatro empresas de telefonia fixa e oito empresas de telefonia móvel (ANATEL, 1998).

Em 2001, o setor de telefonia móvel ficou ainda mais competitivo. A Anatel realizou novamente vendas de licenças para exploração de telefonia móvel, entretanto por meio de novas licitações e mediante um Serviço substituto ao SMC (Serviço Móvel Celular) que passou a ser denominado Serviço Móvel Pessoal (SMP).

Desde então, o SMP apresenta elevado crescimento no Brasil, onde em 2001 o país registrou 28,7 milhões de acesso do serviço móvel e em 2012 os acessos alcançaram a marca de 261,8 milhões, o que torna o Brasil um dos maiores mercados mundiais de telefonia móvel (ANATEL, 2013). 


\subsection{Discussão dos resultados descritivos}

Em função das características heterogêneas nos dados coletados entre as empresas, as variáveis destacadas não apresentaram normalidade, característica necessária para o melhor uso das técnicas de regressão. Neste sentido, todas as variáveis foram padronizadas (razão da diferença de cada evento com a média pelo desvio padrão), Os resultados originais consolidados anualmente de cada variável encontram-se no anexo do artigo.

A Tabela 1 apresenta a matriz de correlação entre as variáveis padronizadas utilizadas na análise de regressão com dados em painel.

Tabela 1- Matriz de correlação

\begin{tabular}{l|c|c|c|c|c|c|c|c|c}
\hline & ROI & ROE & ROS & EBTIDA & VM & EVA $^{\oplus}$ & RROI & Investimento & WACC \\
\hline ROI & 1 & 0,877 & 0,7893 & 0,4634 & 0,029 & 0,5138 & 0,6737 & $-0,0431$ & $-0,4039$ \\
\hline ROE & 0,877 & 1 & 0,884 & 0,42 & 0,1394 & 0,5104 & 0,7356 & $-0,0006$ & $-0,175$ \\
\hline ROS & 0,7893 & 0,884 & 1 & 0,5113 & 0,031 & 0,5378 & 0,6771 & 0,0452 & $-0,139$ \\
\hline EBTIDA & 0,4634 & 0,42 & 0,5113 & 1 & $-0,1879$ & 0,1926 & 0,1214 & $-0,0487$ & $-0,4234$ \\
\hline VM & $\mathbf{0 , 0 2 9}$ & $\mathbf{0 , 1 3 9 4}$ & $\mathbf{0 , 0 3 1}$ & $\mathbf{- 0 , 1 8 7 9}$ & $\mathbf{1}$ & $\mathbf{- 0 , 3 5 2 9}$ & $\mathbf{0 , 2 1 0 2}$ & $\mathbf{0 , 2 6 4 3}$ & $\mathbf{0 , 2 2 4 3}$ \\
\hline EVA & 0,5138 & 0,5104 & 0,5378 & 0,1926 & $-0,3529$ & 1 & 0,7294 & $-0,2219$ & 0,2668 \\
\hline RROI & 0,6737 & 0,7356 & 0,6771 & 0,1214 & 0,2102 & 0,7294 & 1 & 0,0133 & 0,4039 \\
\hline Investimento & $-0,0431$ & $-0,0006$ & 0,0452 & $-0,0487$ & 0,2643 & $-0,2219$ & 0,0133 & 1 & 0,0698 \\
\hline WACC & $-0,4039$ & $-0,175$ & $-0,139$ & $-0,4234$ & 0,2243 & 0,2668 & 0,4039 & 0,0698 & 1 \\
\hline
\end{tabular}

Fonte: Elaborado pelos autores com dados da pesquisa.

Nota-se que os principais coeficientes de correlação encontramse entre as variáveis ROI, ROE e ROS. Para que não haja problemas de multicolinearidade no modelo de regressão, as variáveis de desempenho foram utilizadas em separado.

Verificou-se, ainda, o coeficiente de correlação entre as variáveis de desempenho financeiro com a variação do valor de mercado. Utilizouse a variação do valor de mercado, uma vez que se consegue explicar 
melhor suas oscilações ao longo do tempo do que o valor nominal das empresas. Os dados da matriz encontram-se na Tabela 2. A significância dos coeficientes está associada à rejeição da hipótese nula, sendo esta a ausência de correlação entre as variáveis.

Tabela 2 - Matriz de correlação das variáveis independentes com o VVM

\begin{tabular}{|c|c|c|}
\hline & VVM & p valor \\
\hline ROI & 0,01789213 & 0,8137 \\
\hline ROE & 0,03577173 & 0,6374 \\
\hline ROS & 0,08004058 & 0,291 \\
\hline EBTIDA & $-0,03221089$ & 0,6713 \\
\hline EVA $^{\circledR}$ & $-0,2047362$ & 0,0064 \\
\hline RROI & $-0,02796713$ & 0,7125 \\
\hline Investimento & 0,7315401 & 0 \\
\hline WACC & $-0,05676564$ & 0,4543 \\
\hline
\end{tabular}

Fonte: Elaborado pelos autores com dados da pesquisa.

Observa-se que o maior coeficiente de correlação com a variação do valor de mercado é o investimento, sendo este também significativo. O resultado confirma a teoria, uma vez que o investimento aumenta o valor econômico agregado da empresa $\left(E V A^{\circledR}\right)$, o qual é assumido como uma das principais medidas de criação de valor.

Entretanto, seguindo-se da mesma teoria, observa-se que tanto o RROI como EVA ${ }^{\circledR}$ possuem coeficientes negativos, o que contraria a teoria proposta de criação de valor pelo EVA ${ }^{\circledR}$, de modo que o coeficiente, também foi significativo. O Valor Econômico Agregado é utilizado para indicar se a empresa está criando ou destruindo valor, porém observa-se que a relação encontrada com a variação do valor de mercado demonstra que o crescimento de uma variável ocasiona o decrescimento da outra. 
As variáveis ROI, ROE, ROS e Margem EBTIDA não foram significativas na explicação da VVM, em que pese que os coeficientes sejam positivos exceto para a Margem EBTIDA.

Cabe ressaltar na análise da matriz que os dados foram avaliados individualmente, o que pode indicar uma limitação, uma vez que a VVM não pode ser analisada pelos indicadores isoladamente. Outra ressalva está no fato da análise ser realizada considerando aspectos e desempenhos internos e não incluir fatores externos que podem ter exercido influência no VVM destas empresas no período analisado.

\subsection{Análise regressão com dados em painel}

Em razão das 176 observações (4 empresas x 44 trimestres) optou-se pelo uso de um modelo com três variáveis independentes, além das 4 variáveis dummies relativas às empresas, como forma de garantir maiores graus de liberdade ao modelo além de não "inflar" o $\mathrm{R}^{2}$ com múltiplas variáveis independentes, em especial, as três variáveis de desempenho financeiro que apresentaram elevados coeficientes de correlação.

A partir disso, testou-se o modelo empírico da fórmula 12 alternando combinações com todas as variáveis, no interesse de alcançar o objetivo do trabalho.

Os resultados das combinações foram semelhantes, de modo que se reproduziu aqui os resultados previstos no modelo teórico (12) que foram significativos, conforme Tabela 3. 
Tabela 3 - Resultados da regressão em painel. Variável dependente: variação do valor de mercado.

\begin{tabular}{|c|c|c|c|}
\hline \multirow{4}{*}{ Modelo 1} & Variáveis & Coeficientes & Intervalo de Confiança \\
\hline & $\begin{array}{l}\text { Constante } \\
\text { Investimento } \\
\text { WACC } \\
\text { ROI }\end{array}$ & $\begin{array}{c}0,00456223 \\
0,75538^{* *} \\
-0,120597^{*} \\
-0,0121988\end{array}$ & $\begin{array}{c}(-0,0957698 ; 0,104894) \\
(0,652560 ; 0,858200) \\
(-0,258964 ; 0,0177699) \\
(-0,127650 ; 0,103252)\end{array}$ \\
\hline & Testes & Estatística & p valor \\
\hline & $\begin{array}{l}\mathrm{R}^{2} \\
\text { Durbin Watson } \\
\text { Estatística F } \\
\text { Heteroscedasticidade } \\
\text { Normalidade } \\
\text { Teste para diferenciar intercepto do grupo }\end{array}$ & $\begin{array}{c}0,56301 \\
1,981509 \\
36,28942 \\
2,28882 \\
143,365 \\
2,07929\end{array}$ & $\begin{array}{l}0,431734 \\
4,97 \mathrm{E}-28 \\
0,682805 \\
7,39 \mathrm{E}-32 \\
0,104832\end{array}$ \\
\hline \multirow{4}{*}{ Modelo 2} & Variáveis & Coeficientes & Intervalo de Confiança \\
\hline & $\begin{array}{l}\text { Constante } \\
\text { Investimento } \\
\text { WACC } \\
\text { ROE }\end{array}$ & $\begin{array}{c}0,00456223 \\
0,755045^{\star * *} \\
-0,115026^{*} \\
0,00247887\end{array}$ & $\begin{array}{c}(-0,0957821 ; 0,104907) \\
(0,652124 ; 0,857966) \\
(-0,245273 ; 0,0152215) \\
(-0,104124 ; 0,109082)\end{array}$ \\
\hline & Testes & Estatística & $\mathrm{p}$ valor \\
\hline & $\begin{array}{l}\mathrm{R}^{2} \\
\text { Durbin Watson } \\
\text { Estatística F } \\
\text { Heteroscedasticidade } \\
\text { Normalidade } \\
\text { Teste para diferenciar intercepto do grupo }\end{array}$ & $\begin{array}{c}0,562903 \\
1,975113 \\
36,27363 \\
2,24149 \\
140,026 \\
2,0309\end{array}$ & $\begin{array}{l}0,413466 \\
5,08 \mathrm{E}-28 \\
0,691442 \\
3,92 \mathrm{E}-31 \\
0,111461\end{array}$ \\
\hline \multirow{4}{*}{ Modelo 3} & Variáveis & Coeficientes & Intervalo de Confiança \\
\hline & $\begin{array}{l}\text { Constante } \\
\text { Investimento } \\
\text { WACC } \\
\text { ROS }\end{array}$ & $\begin{array}{c}0,00456223 \\
0,753244^{* * *} \\
-0,111009^{*} \\
0,0287191\end{array}$ & $\begin{array}{c}(-0,0956929 ; 0,104817) \\
(0,650296 ; 0,856192) \\
(-0,241152 ; 0,0191327) \\
(-0,0742500 ; 0,131688)\end{array}$ \\
\hline & Testes & Estatística & p valor \\
\hline & $\begin{array}{l}\mathrm{R}^{2} \\
\text { Durbin Watson } \\
\text { Estatística F } \\
\text { Heteroscedasticidade } \\
\text { Normalidade } \\
\text { Teste para diferenciar intercepto do grupo }\end{array}$ & $\begin{array}{c}0,56368 \\
1,964706 \\
36,38842 \\
2,19884 \\
134,021 \\
2,04304\end{array}$ & $\begin{array}{l}0,387088 \\
4,38 \mathrm{E}-28 \\
0,699242 \\
7,90 \mathrm{E}-30 \\
0,109761\end{array}$ \\
\hline \multirow{4}{*}{ Modelo 4} & Variáveis & Coeficientes & Intervalo de Confiança \\
\hline & $\begin{array}{l}\text { Constante } \\
\text { Investimento } \\
\text { WACC } \\
\text { EBTIDA }\end{array}$ & $\begin{array}{l}0,00456223 \\
0,753723^{* * *} \\
-0,139217^{*} \\
-0,0432752 \\
\end{array}$ & $\begin{array}{c}(-0,0956121 ; 0,104737) \\
(0,651019 ; 0,856427) \\
(-0,282407 ; 0,00397338) \\
(-0,155819 ; 0,0692683)\end{array}$ \\
\hline & Testes & Estatística & p valor \\
\hline & $\begin{array}{l}\mathrm{R}^{2} \\
\text { Durbin Watson } \\
\text { Estatística F } \\
\text { Heteroscedasticidade } \\
\text { Normalidade } \\
\text { Teste para diferenciar intercepto do grupo }\end{array}$ & $\begin{array}{c}0,564383 \\
1,991196 \\
36,49254 \\
2,09587 \\
142,044 \\
1,99001 \\
\end{array}$ & $\begin{array}{l}0,455628 \\
3,83 \mathrm{E}-28 \\
0,718131 \\
1,43 \mathrm{E}-31 \\
0,117379\end{array}$ \\
\hline
\end{tabular}

Significância: *** $99 \%,{ }^{*} 90 \%$.

Fonte: Elaborado pelos autores com dados da pesquisa. 
Pode-se observar que, nos quatro modelos, somente foram significativos o Investimento e o WACC para a explicação do valor de mercado com significância de $99 \%$ e 90\%, respectivamente. Entre os indicadores de desempenho financeiro, o ROE apresentou o menor coeficiente, indicando ser o que menos influencia a variação do valor de mercado.

O EBTIDA apesar de apresentar o maior coeficiente, ainda é um valor muito pequeno, e dessa forma não pode ser considerado um fator relevante. Esse resultado confirma o estudo de Paiva, Savoia e Sousa (2008), no qual, entre outras variáveis dependentes, a Margem EBTIDA e o ROI também não foram significativos na explicação da variação do valor de mercado.

Apesar do ROE e o ROS exibirem coeficientes positivos, nota-se que nenhum dos intervalos de confiança desses indicadores encontrase somente entre valores positivos. O ROI, assim como o EBTIDA, contraria a teoria ao apresentar coeficiente negativo, no entanto uma parcela do seu intervalo de confiança encontra-se em valores positivos.

O Investimento e o WACC são os indicadores que exercem influência na variação do valor de mercado. Entretanto, apesar de eles estarem contidos no cálculo do EVA ${ }^{\circledR}$, o Valor Econômico Agregado não apresentou significância e, portanto não foi utilizado entre os modelos e o resultado do modelo foi muito próximo ao observado com a variável ROI. Tal relação do EVA ${ }^{\circledR}$ com valor de mercado contradiz o estudo de O'Byrne (1996), um dos primeiros sobre o tema, bem como os resultados de Araújo e Assaf Neto (2003), no entanto é confirmado pelo estudo mais recente de Bastos et al. (2009).

Os testes realizados indicam os ajustes estatísticos do modelo. $\mathrm{O}$ $\mathrm{R}^{2}$ é considerado adequado à medida que a capacidade das variáveis em explicar a variação de valor de mercado em todos os casos foi superior a $40 \%$. Também se mostra adequada a estatística F em apresentar p-valor próximo de zero, sendo a hipótese nula que os coeficientes são nulos, ou seja, não significativos para explicar a variável dependente. $O$ teste de Durbin Watson demonstra que não há, em nenhum dos quatro modelos, 
autocorrelação entre as variáveis e os resíduos, pois p-valor é maior 5\% sendo a hipótese nula a ausência de autocorrelação.

O teste de heterocedasticidade também confirma o ajuste apropriado dos modelos por indicar que os termos de erro das regressões apresentam a mesma variância, ou seja, possuem características homocedásticas, pois o p-valor é superior a $5 \%$, sendo a hipótese nula a distribuição homocedástica do termo de erro.

Realizou-se teste para diferenciar os interceptos de cada empresa, e assinala-se que apesar de apresentarem valores distintos, estes não foram significativos, ou seja, os coeficientes encontrados podem ser analisados para todo o grupo. Apenas o teste de normalidade dos resíduos não é adequado ao indicar que o erro não possui distribuição normal, essa restrição impossibilita o uso dos resultados para fins inferência, porém entende-se que ela não invalida o modelo, em função dos resultados observados na Tabela 2 de correlação das variáveis independentes com a variação do valor de mercado e a própria constância dos resultados das duas variáveis significativas.

\section{Considerações finais}

A proposta deste trabalho foi analisar o relacionamento entre o desempenho financeiro e o valor de mercado das empresas de telefonias instaladas no Brasil. A amostra utilizada computou quatro grandes empresas do setor com dados disponíveis na BM\&FBOVESPA entre 2002 e 2012.

A abordagem metodológica utilizada respondeu ao objetivo do trabalho identificando os indicadores que mais influenciam o valor de mercado das empresas de telefonia, bem como avaliou o grau de importância de cada um deles. Para análise de regressão foram propostos quatro modelos cuja variação ocorreu somente com os indicadores de desempenho ROE, ROS e ROI, além do EBTIDA, mantendo-se fixo o Investimento e WACC. Em todos os casos analisados, somente o 
Investimento e o WACC mostraram-se significativos na explicação da variação do valor de mercado.

O motivo de o WACC apresentar valores mais similares entre as empresas, ter sido mais significativo seria em função das margens de retorno restritas despertando a atenção dos investidores quanto às condições dos financiamentos e a estrutura de capital assumida. Já para o investimento, sugere-se para a contínua necessidade de renovação de ativos, frente ao déficit ainda existente na infraestrutura de telecomunicações nacional em relação ao mundo, de modo que, assumir determinadas tecnologias primeiramente (first mover) pode ser um fator importante de sucesso.

Com a ressalva de que os dados analisados consideraram apenas aspectos internos à organização e desconsiderando fatores externos que interferem na variação do valor de mercado, pode-se concluir que, para o caso estudado, os indicadores de desempenho ROE, ROS, ROI e EBTIDA não foram determinantes no valor de mercado das empresas. Dessa forma, para o setor de telefonia, ao Investimento e ao WACC devem ser atribuídos grande relevância para a variação do valor de mercado das empresas.

Em tempo, é preciso considerar as limitações do trabalho como o tamanho da amostra de apenas quatro empresas, a restrição temporal de dez anos devido à ausência de dados que contemplem todas as empresas da amostra em anos anteriores e, principalmente, a ausência de normalidade dos resíduos nos modelos da regressão mesmo após a padronização dos dados.

Espera-se, a partir deste estudo, que sejam desenvolvidas outras pesquisas sobre o valor de mercado e desempenho financeiro para que sejam esclarecidos os melhores indicadores que influenciam o valor de mercado de empresas atuantes em setores distintos, além de pesquisas que possam complementar o trabalho, estudando os fatores externos à empresa que sejam determinantes na variação do valor de mercado. 


\section{Referências}

ALVIM, M A.; COSTA, L. G. T. A.; COSTA, L. R. T. A. Valuation: Manual de avaliação e reestruturação econômica de empresas. 2. ed. São Paulo: Atlas, 2010.

AGÊNCIA NACIONAL DE TELECOMUNICAÇÕES. Ato $n^{\circ}$ 673/1998, 03 de agosto de 1998. Brasília, DF. Disponível em: <http://www.anatel. gov.br/Portal/verificaDocumentos/documento.asp?numeroPublicacao= 6991\&assuntoPublicacao=null\&caminhoRel=null\&filtro=1\&documentoP ath=biblioteca/atos/1998/ato_673_1998.pdf>. Acesso em: 11 nov. 2012.

. Relatório Anual da Anatel 2001. Brasília, DF. Disponível em: <http://www.anatel.gov.br/Portal/verificaDocumentos/documento.asp?n umeroPublicacao $=50017 \&$ assuntoPublicacao $=$ null\&caminhoRel=null \& filtro=1\&documentoPath=biblioteca/publicacao/relatorio_anatel_2001. pdf>. Acesso em: 11 nov. 2012.

Relatório Anual da Anatel 2013. Brasília, DF. Disponível em: <http://www.anatel.gov.br/Portal/verificaDocumentos/documento.asp?n umeroPublicacao $=312603 \&$ assuntoPublicacao $=$ null \& caminhoRel=null \& filtro=1\&documentoPath=312603.pdf>. Acesso em: 24 jul. 2013.

ARAÚJO, A. M. P.; ASSAF NETO, A. A Contabilidade Tradicional e a Contabilidade Baseada em Valor. Revista Contabilidade \& Finanças USP, São Paulo, v. 14, n. 33, p. 16-32, set./dez. 2003.

ARAÚJO, E. A. T.; OLIVEIRA, V. C.; SILVA, W. A. C. CAPM em estudos brasileiros: uma análise da pesquisa. Revista de Contabilidade e Organizações, Ribeirão Preto-SP, v. 6, n. 15, p. 95-122, maio/ago. 2012.

ASSAF NETO, A. Finanças Corporativas e Valor. 2 ed. São Paulo: Atlas, 2005.

BARCELLOS, E. P. Internacionalização de Empresas Brasileiras: um estudo sobre a relação entre o grau de internacionalização e desempenho financeiro. 2010. 177f. Dissertação (Mestrado) Faculdade de Economia e Administração, Universidade de São Paulo, São Paulo, 2010. 
BASSO, L. F. C.; ALVES, W.; NAKAMURA, W. T. Medidas de valor adicionado: um estudo do impacto das dificuldades encontradas para a estimativa do custo total de capital na opção pela utilização deste tipo de medida em empresas operando no Brasil. In: ENCONTRO BRASILEIRO DE FINANÇAS, 2., 2002, Rio de Janeiro. Anais... Rio de Janeiro: IBMEC-RJ, 2002. p. 1-16. Disponível em: <http://bibliotecadigital.fgv.br/ ocs/index.php/ebf/2EBF>. Acesso em: 10 ago. 2012.

BASSO, L. F. C. (Org.) EVA : Gestão Baseada em Valor. New York: McGraw-Hill, 2003.

BASTOS, D. D., NAKAMURA, W. T., DAVID, M., ROTTA, U. A. S. A relação entre o retorno das ações e as métricas de desempenho: evidências empíricas para as companhias abertas no Brasil. Revista Gestão USP, São Paulo, v. 16, n. 3, p. 65-79, jul./set. 2009.

BIDDLE, G.; BOWEN, R.; WALLACE, J. Does EVA® beat earnings? Evidence on associations with stock returns and firm values. Journal of Accounting and Economics, Massachusetts, v. 24, n. 3, p. 301-336, dec. 1997.

BRITO, E. P. Z., BRITO, L. A. L., MORGANTI, F. Inovação e Desempenho Empresarial: Lucro ou Crescimento? Revista de Administração de Empresas, São Paulo, v. 8, n. 1, p. 1-24, jan./jun. 2009.

CAIXE, D. F. Relação Dinâmica entre a estrutura de propriedade e controle e o valor de mercado corporativo no Brasil: análise da primeira década do século XXI. 2012. 138f. Dissertação (Mestrado) Faculdade de Economia e Administração, Universidade de São Paulo, Ribeirão Preto-SP, 2012.

CUÉLLAR-FERNANDÉZ, B.; FUERTES-CALLÉN, Y.; LAÍNEZGADEA, J. A. The impact of strategic alliances on the market value of telecommunications firms. Journal of high technology management research, Amsterdam , v. 22, n. 1, p. 1-13, 2011.

DAMODARAN, A. Corporate Finance: theory and practice. 2 nd. [S.I]: Wiley series in finance, 2001. 
DENARDIN, A. A. A importância do Custo de Oportunidade para a avaliação de empreendimentos baseados na criação de valor econômico (Economic Value Added - EVA). Revista Contexto - UFRGS, Porto Alegre, v. 4, n. 6, p. 1-20, jan./jun. 2004.

GITMAN, L. J. Princípios de administração financeira. 10 ed. São Paulo: Pearson, 2004.

GODOY, J. A. R.; MORALES, D. S. A. El cargo de capital en la evaluación del desempeño financeiro de empresas innovadoras de confecciones de Cali. Estudios Gerenciales, Cali, v. 28, n. 123, p. 85-100, abr./jun., 2012.

GONTHIER, H. F. A. Qualidade nas Telecomunicações. 2003. 122f. Dissertação (Mestrado) - Faculdade de Engenharia Mecânica, Universidade Estadual de Campinas. Campinas, 2003.

GRAHAM, J. R.; HARVEY, C. R. The theory and practice of corporate finance: evidence from the field. Journal of Financial Economics, Newarks, v. 60, n. 2-3, p. 187-243, maio 2001.

GUJARATI, D. N. Econometria Básica. 4 ed. Rio de Janeiro: Elsevier, 2006.

JUNG, Hyung-I1. WACC as the touchstone performance indicator The use of financial ratios as performance indicator - from operations to capital investments. International Journal of Contemporary Hospitality Management, Reino Unido, v. 20, n. 6, p. 700-710, 2008.

KAYO, E. K., KIMURA, H., MARTIN, D. M. L., NAKAMURA, W. T. Ativos Intangíveis, Ciclo de Vida e Criação de Valor. Revista de Administração Contemporânea, Curitiba-PR, v. 10, n, 3, p. 73-90, jul./set. 2006.

KOCHE, J. C. Fundamentos de Metodologia Científica: teoria da ciência e iniciação à pesquisa. 30 ed., Petrópolis: Vozes, 2012.

KRAUTER, E. Medidas de avaliação de desempenho e criação de valor. In: SEMINÁRIOS DE ADMINISTRAÇÃO FEA/USP, 7., 2004, São Paulo. 
Anais... São Paulo: USP, 2004. p. 1-16. Disponível em: <http://www. ead.fea.usp.br/Semead/7semead/>. Acesso em: 10 ago. 2012.

KRAUTER, E.; SOUSA, A. F. Medidas de avaliação de desempenho financeiro e criação de valor para o acionista: um estudo de caso. In: SEMINÁRIOS DE ADMINISTRAÇÃO FEA/USP, 10., 2007, São Paulo. Anais... São Paulo: USP, 2007. p. 1-16. Disponível em: <http://www.ead. fea.usp.br/semead/10semead/sistema/resultado/trabalhosPDF/519. pdf>. Acesso em: 10 ago. 2012.

LEHN, K., MAKHIJA, A. K. EVA \& MVA as performance measures and signals for strategic change. Strategy \& Leadership, United Kingdom (Reino Unido), v. 24, n. 3, p. $34-38,1996$.

LEITE, T. S., SANTOS, D. F. L. A Relação dos Ativos Intangíveis e o Valor de Mercado na Indústria de Materiais Básicos do Brasil. Revista Brasileira de Administração Científica, Aquidauana-SE, v. 4, n. 1, p. 104-121, jan./jun. 2013.

LI, F.; WHALLEY, J. Deconstruction of the telecommunications industry: from value chains to value networks. Telecommunications Policy, Göteborg, Sweden, v. 26, n. 9-10, p. 451-472, oct./ nov. 2002.

MEDEIROS, O. R. Empirical evidence on the relationship between EVA and Stock Returns in Brazilian firms. Revista Contabilidade, Gestão e Governança, Brasília-DF. v. 12, n. 1, p. 3-9, jan./abr. 2009.

O'BYRNE, S. F. EVA® and Market Value. Journal of Applied Corporate Finance, Malden MA,v. 9, n. 1, p. 116-125, Spring 1996.

PAIVA, E. V. S., SAVOIA, J. R. F., SOUSA, A. F. Impacto de medidas de avaliação de desempenho financeiro no valor das ações. In: SEMINÁRIOS DE ADMINISTRAÇÃO FEA/USP, 11., 2008, São Paulo. Anais... São Paulo: USP, 2008.p. 1-14. Disponível em: <http://www. ead.fea.usp.br/Semead/11semead/resultado/trabalhosPDF/286.pdf>. Acesso em: 12 dez. 2012. 
PEREZ, M. M.; FAMÁ, R. Ativos intangíveis e o desempenho empresarial. Revista de Contabilidade \& Finanças - USP, São Paulo, n. 40, p. 7-24, jan./abr. 2006.

QUINTELLA, R. H., COSTA, M. A. O setor de Telefonia Móvel do Brasil após o SMP: as Estratégias das operadoras e a convergência fixamóvel. Revista de Administração Púbica, Rio de Janeiro, v. 43, n. 1, p. 123-150, jan./fev. 2009.

ROSS, S. A; WESteRfIELD, R. W., JORDAN, B. D. Princípios de administração financeira. 2 ed. São Paulo: Atlas, 2008.

SALVI, A. A relação do retorno das ações com o EVA®, com o lucro residual e com as medidas contábeis tradicionais: um estudo empírico aplicado às empresas brasileiras de capital aberto. Ribeirão Preto-SP, 2007. 93f. Dissertação (Mestrado) - Faculdade de Economia e Administração, Universidade de São Paulo, 2007.

SRINIVASAN, S.; HANSSENS, D. M. Marketing and Firm Value: Metrics, Methods, Findings, and Future Directions. Journal of Marketing Research, Chicago, v. 46, n. 3, p. 293-312, jun. 2009.

STEWART III, G. B. The quest for value: the EVA® management guide. New York: Harper Business, 1990.

WHEATLEY, J. J. Performance measures in telecommunications: changing needs. Technovation, England, v. 8, n. 1-3, chapter 12, p. 201-216, 1988.

XIN, Z.; TING, W.; YUAN, Z. Economic Value Added for Performance Evaluation: a Financial Engineering. Systems Engineering Procedia, Toronto, v. 5, p. 379-387, 2012. 


\section{ANEXO}

\begin{tabular}{|c|c|c|c|c|c|c|c|c|c|c|}
\hline Empresa & Ano & ROI & ROE & ROS & EBTIDA & VM & EVA & RROI & Investimento & WACC \\
\hline \multirow{11}{*}{ TIM } & 2002 & 6,90 & 6,45 & 8,83 & 35,70 & $1.053 .060 .166,25$ & $-20.991 .820 .621,46$ & $-19,71$ & $1.073 .942 .500,00$ & 26,61 \\
\hline & 2003 & 8,68 & 9,13 & 11,60 & 33,70 & $885.429 .125,22$ & $-18.346 .584 .387,08$ & $-20,08$ & $902.735 .750,00$ & 28,75 \\
\hline & 2004 & 14,50 & 12,83 & 13,10 & 27,90 & $1.840 .833 .981,43$ & $-11.265 .434 .084,34$ & $-8,96$ & $1.352 .965 .750,00$ & 23,46 \\
\hline & 2005 & 19,38 & 16,23 & 13,65 & 34,60 & $3.184 .860 .922,55$ & $-17.131 .432 .767,45$ & $-8,78$ & $1.931 .690 .750,00$ & 28,15 \\
\hline & 2006 & 2,48 & $-2,48$ & $-1,20$ & 24,70 & $19.706 .305 .189,50$ & $-669.586 .197 .094,96$ & $-66,99$ & $10.023 .819 .250,00$ & 69,46 \\
\hline & 2007 & 3,05 & $-0,03$ & $-0,05$ & 23,78 & $20.036 .684 .485,50$ & $-144.293 .031 .499,67$ & $-14,76$ & $9.774 .515 .500,00$ & 17,81 \\
\hline & 2008 & 2,78 & 0,48 & 0,30 & 21,65 & $13.564 .440 .307,50$ & $-182.590 .755 .950,29$ & $-18,08$ & $10.088 .386 .000,00$ & 20,85 \\
\hline & 2009 & 3,13 & 2,33 & 1,35 & 23,08 & $13.957 .043 .000,00$ & $-189.825 .953 .119,02$ & $-17,36$ & $10.900 .543 .750,00$ & 20,49 \\
\hline & 2010 & 5,33 & 10,85 & 6,78 & 27,38 & $16.401 .333 .260,00$ & $-137.560 .421 .656,16$ & $-11,38$ & $12.094 .460 .250,00$ & 16,70 \\
\hline & 2011 & 8,93 & 22,43 & 14,25 & 27,95 & $21.274 .781 .021,50$ & $-117.771 .118 .036,20$ & $-8,55$ & $13.808 .050 .000,00$ & 17,48 \\
\hline & 2012 & 9,95 & 11,00 & 7,50 & 26,73 & $24.905 .226 .920,25$ & $-101.094 .996 .550,98$ & $-6,15$ & $16.322 .049 .250,00$ & 16,10 \\
\hline \multirow{11}{*}{ VIVO } & 2002 & 4,63 & 4,10 & 9,50 & 48,80 & $18.994 .257 .456,00$ & $-406.458 .512 .690,52$ & $-20,79$ & 19.536 & 25,41 \\
\hline & 2003 & 5,45 & 5,80 & 10,33 & 45,30 & 19.283 .98 & -351. & $-20,17$ & 17.2 & 25,62 \\
\hline & 2004 & 8,35 & 10,30 & 14,70 & 45,70 & $25.081 .764 .602,00$ & $-174.435 .331 .605,10$ & $-11,31$ & $15.353 .993 .250,00$ & 19,66 \\
\hline & 2005 & 11,10 & 13,95 & 16,63 & 45,80 & $23.279 .541 .856,75$ & $-157.596 .401 .156,89$ & $-11,55$ & $13.370 .480 .000,00$ & 22,65 \\
\hline & 2006 & 12,98 & 16,15 & 18,08 & 47,30 & $25.503 .759 .942,75$ & $-76.438 .913 .781,51$ & $-5,81$ & $13.376 .343 .750,00$ & 18,79 \\
\hline & 2007 & 11,63 & 13,83 & 15,98 & 43,10 & $28.801 .313 .824,00$ & $-55.072 .913 .317,29$ & $-4,18$ & $13.199 .269 .750,00$ & 15,80 \\
\hline & 2008 & 11,53 & 14,18 & 14,28 & 40,70 & $23.525 .617 .171,25$ & $-56.283 .279 .789,59$ & $-4,25$ & $13.178 .396 .000,00$ & 15,77 \\
\hline & 2009 & 10,80 & 12,95 & 13,20 & 37,65 & $23.535 .068 .277,50$ & $-16.781 .063 .867,23$ & $-1,17$ & $13.223 .013 .250,00$ & 11,97 \\
\hline & 2010 & 11,40 & 13,53 & 14,03 & 34,50 & $19.836 .688 .929,25$ & $-14.940 .038 .423,52$ & $-1,00$ & $13.396 .435 .750,00$ & 12,40 \\
\hline & 2011 & 6,98 & 9,00 & 13,15 & 33,78 & $46.843 .423 .074,75$ & $-346.609 .440 .358,83$ & $-8,59$ & $41.901 .644 .750,00$ & 15,57 \\
\hline & 2012 & 5,43 & 5,95 & 12,20 & 35,73 & $56.335 .435 .000,00$ & $-300.044 .036 .359,58$ & $-5,80$ & $51.602 .184 .250,00$ & 11,22 \\
\hline \multirow{11}{*}{ EMBRATEL } & 2002 & 0,00 & $-12,90$ & $-9,80$ & 22,10 & 5.44 & -272 & $-28,28$ & 1,00 & 28,28 \\
\hline & 2003 & 2,33 & $-1,73$ & $-1,03$ & 24,30 & $6.007 .505 .720,15$ & $-197.956 .708 .206,38$ & $-21,72$ & 9.002 .036 .0 & 24,04 \\
\hline & 2004 & 4,50 & $-0,80$ & 0,03 & 19,60 & $6.242 .581 .385,68$ & $-137.515 .124 .171,30$ & $-16,47$ & $8.398 .324 .750,00$ & 20,97 \\
\hline & 2005 & 3,70 & $-1,53$ & $-0,40$ & 23,20 & $5.186 .532 .560,98$ & $-178.203 .967 .454,18$ & $-21,45$ & 8.347.169.250,00 & 25,15 \\
\hline & 2006 & 3,55 & 2,13 & 2,48 & 15,70 & $7.970 .348 .537,38$ & $-184.768 .578 .030,85$ & $-18,72$ & $9.852 .780 .500,00$ & 22,27 \\
\hline & 2007 & 5,23 & 7,43 & 7,23 & 29,00 & $8.800 .585 .732,73$ & $-148.320 .090 .384,48$ & $-13,99$ & $10.665 .596 .000,00$ & 19,22 \\
\hline & 2008 & 6,95 & 8,43 & 7,93 & 23,40 & $11.529 .666 .005,25$ & $-127.613 .725 .665,12$ & $-11,25$ & $11.295 .910 .000,00$ & 18,20 \\
\hline & 2009 & 8,18 & 10,45 & 9,38 & 29,10 & $13.155 .939 .649,00$ & $-68.514 .946 .514,74$ & $-5,84$ & $11.736 .120 .500,00$ & 14,02 \\
\hline & 2010 & 8,28 & 10,15 & 9,48 & 30,53 & $14.272 .736 .856,75$ & $-92.794 .349 .900,63$ & $-6,65$ & $13.425 .962 .000,00$ & 14,92 \\
\hline & 2011 & 5,63 & 5,45 & 5,18 & 29,55 & $16.478 .921 .599,75$ & $-290.514 .361 .507,32$ & $-15,78$ & $18.382 .205 .500,00$ & 21,41 \\
\hline & 2012 & 6,00 & 5,10 & 3,95 & 27,93 & $17.527 .416 .823,25$ & $-170.343 .819 .967,31$ & $-7,40$ & $23.193 .573 .500,00$ & 13,40 \\
\hline \multirow{11}{*}{ 이 } & 2002 & 3,90 & 3,33 & 4,93 & 47,20 & $9.713 .127 .886,43$ & $-170.427 .297 .730,53$ & $-14,31$ & $11.910 .421 .500,00$ & 18,21 \\
\hline & 2003 & 3,78 & 2,10 & 4,23 & 37,50 & $10.560 .585 .106,83$ & $-191.976 .671 .324,82$ & $-15,78$ & $12.163 .724 .250,00$ & 19,56 \\
\hline & 2004 & 3,58 & 3,10 & 4,25 & 39,20 & $9.611 .577 .497,48$ & $-137.121 .835 .637,77$ & $-12,06$ & $11.370 .492 .500,00$ & 15,64 \\
\hline & 2005 & 0,88 & $-1,25$ & $-0,53$ & 31,43 & $10.391 .293 .216,55$ & $-189.324 .553 .551,57$ & $-16,67$ & $11.378 .742 .000,00$ & 17,55 \\
\hline & 2006 & 2,78 & 3,35 & 2,30 & 33,65 & $11.666 .199 .723,75$ & $-112.105 .493 .138,85$ & $-10,66$ & $10.466 .861 .500,00$ & 13,43 \\
\hline & 2007 & 5,65 & 8,88 & 7,35 & 35,38 & $14.875 .315 .028,00$ & $-50.999 .094 .897,02$ & $-5,21$ & $9.745 .568 .500,00$ & 10,86 \\
\hline & 2008 & 7,23 & 12,13 & 10,55 & 34,85 & $19.834 .164 .690,75$ & $-56.028 .995 .483,21$ & $-5,15$ & $11.120 .667 .000,00$ & 12,37 \\
\hline & 2009 & $-6,30$ & $-14,20$ & $-14,18$ & 0,50 & $18.429 .098 .156,25$ & $-238.680 .827 .253,84$ & $-15,98$ & $15.011 .491 .250,00$ & 9,68 \\
\hline & 2010 & 5,70 & 9,83 & 16,00 & 33,25 & $8.710 .102 .962,30$ & $-89.027 .145 .577,50$ & $-5,02$ & $17.655 .723 .750,00$ & 10,72 \\
\hline & 2011 & 4,05 & 5,55 & 9,25 & 29,25 & $8.899 .086 .732,50$ & $-158.059 .417 .100,95$ & $-9,09$ & $17.357 .698 .500,00$ & 13,14 \\
\hline & 2012 & 5,45 & 9,83 & 8,48 & 30,88 & $35.842 .280 .940,00$ & $-483.262 .893 .807,36$ & $-10,27$ & $46.729 .568 .500,00$ & 15,72 \\
\hline
\end{tabular}

\section{Artigo recebido em: 16/01/2015 Aprovado em: 04/05/2015}

